\title{
Ionising photon budget in galaxies
}

\author{
Göran Östlin and the LARS team \\ Department of Astronomy \& The Oskar Klein Centre, Stockholm University, AlbaNova \\ University Center, SE 10691 Stockholm. \\ email: ostlin@astro.su.se
}

\begin{abstract}
Understanding the production of ionising photons in galaxies, how these photons propagate, ionise and heat the interstellar medium and possibly leak out of their host galaxies is key to understand many aspects of galaxy evolution and their contribution to reionisation. I will outline some projects currently undertaken to investigate the ionising photon budget.
\end{abstract}

Keywords. Galaxy evolution, Reionisation, Star formation in galaxies.

\section{Introduction}

Massive star formation is tightly connected to the production of photons with energy higher than the hydrogen ionisation potential. Such photons are referred to as ionising photons or alternatively as Lyman continuum (LyC) photons since they have wavelengths shorter than the Lyman limit $(\lambda<912 \AA)$. Ionising photons heat and change the structure of the interstellar medium (ISM) in galaxies, and are the main power source of the nebular spectrum observed in star forming galaxies. The LyC photon energy is reemitted as hydrogen recombination lines and as nebular continuum. These lines (e.g. Ly $\alpha$ and $\mathrm{H} \alpha$ ) are in turn commonly used to survey galaxies, infer their star formation rate, redshift, dust reddening etc. Collisionally excited states of ionised heavy ions furthermore allows the determination of other physical quantities like the density, temperature, ionisation structure and metallicity of the ionised gas.

Depending on the structure of the ISM the LyC photons travel some distance before they ionise, and a fraction of the photons do not lead to ionisation of the ISM but are absorbed by dust or may leak out of the galaxy, either because the galaxy is density bounded (there are enough LyC photons to ionise the entire nebula) or because the ISM is clumpy/porous in which case there may exist optically thin paths from the source of LyC photons (e.g. a young stellar cluster) out of the galaxy. Leaking $\mathrm{LyC}$ from galaxies is likely to have contributed to the reionisation of the universe, and in the local universe they still contribute to keeping the Interagalactic medium ionised. Hence, hydrogen recombination lines trace the propagation of LyC photons and the ionising budget in galaxies.

\section{The Lyman Alpha Reference Sample(s)}

The Ly $\alpha$ line is one of the main spectroscopic probes of the high redshift galaxy population. It is a resonance line and, just like $\mathrm{LyC}$ photons, sensitive to the density and distribution of the neutral ISM (and in addition its kinematics). The Lyman Alpha Reference Sample (LARS, Östlin et al. 2014) has been designed to study the propagation of Ly $\alpha$ photons in local galaxies akin of high-z Lyman Break Galaxies (LBGs) and Ly $\alpha$ Emitters (LAEs). The backbone of LARS is 8 filter HST imaging from the far UV to the i-band and Ly $\alpha, \mathrm{H} \alpha, \mathrm{H} \beta$; plus COS far UV spectroscopy that samples the Ly $\alpha$ line shape and metallic absorption features from the neutral ISM. To this we add ground 
based IFU spectroscopy (with ESO/VLT/MUSE and CAHA/PMAS), Hi imaging (with JVLA and the GMRT) and far IR and molecular line observations. The total sample of LARS and its extensions is now closing in on 50 galaxies and still counting. Hence, this sample is very fit to investigate the ionising budget in actively star forming galaxies.

LARS utilises multicomponent SED fitting and for each spatial resolution element determines the age and mass of the young population and the number of LyC photons locally produced. This can be used to create a synthetic $\mathrm{H} \alpha$ image, which when accounting for the nebular reddening can be compared to the observed $\mathrm{H} \alpha$ image. The ionising budget is sensitive to the massive star content, e.g. stellar rotation (Leitherer et al. 2014) and the upper mass limit of the IMF, which can therefore in principle be constrained. One can also combine this with IFU spectroscopy where certain line ratios (e.g. [OIII]/[SII]) can be used to infer the optical depth of LyC radiation (Pellegrini et al. 2012).

An interesting case is Tol1247-232 which is a rare example of a galaxiy with a direct detection of leaking LyC radiation on the order of a few percent (FUSE observations reported in Leitet et al. 2013). Our COS spectroscopy confirms the leakage (Puschnig et al. in prep.) and locates it to the central region with two very bright star clusters. These clusters have an age of $3 \mathrm{Myr}$ for which the precited $\mathrm{H} \alpha$ equivalent width is $W(\mathrm{H} \alpha)=1600 \AA$. If $\mathrm{LyC}$ radiation is leaking out we would expect a reduced $W(\mathrm{H} \alpha)$ but a $\mathrm{Ly} \alpha / \mathrm{H} \alpha$ ratio close to case B recombination. The observed equivalent width is $\sim 200 \AA$ and $\mathrm{Ly} \alpha / \mathrm{H} \alpha$ $\sim 10$ indicating that ionising photons leak out, but a fraction may also travel inside the galaxy before they ionise. Examining the synthetic $\mathrm{H} \alpha$ image reveals that we only recover $20 \%$ of the ionising budget for the central clusters, while for larger radii it asymptotically reaches $95 \%$ for $r>4 \mathrm{kpc}$. Hence, the ionising budget suggests LyC leakage.

Verhamme et al. (2015) has modelled the Ly $\alpha$ profile for two scenarios of LyC leakage: a homogeneous medium optically thin to LyC (predicting a separation between the blue and red Ly $\alpha$ emission bumps of less than $300 \mathrm{~km} / \mathrm{s}$ ) and an extremely clumpy ISM with clear paths through which LyC can escape (which should produce Ly $\alpha$ emission at the rest wavelength). The $\operatorname{COS} \operatorname{Ly} \alpha$ spectrum shows a redshifted main component and a blue peak (separated by $500 \mathrm{~km} / \mathrm{s}$ ), but no emission at line center. These observations are at odds with the study by Verhamme et al. (2015). The analysis of ISM absorption lines reveal a very clumpy medium with low covering fraction of neutral gas but a high covering of highly ionised gas. The observations can be reconciled with the predictions for models if the interclump column densities are higher than the optical thickness $(\tau=1)$ limit for $\operatorname{Ly} \alpha\left(\sim 10^{13} \mathrm{~cm}^{-1}\right)$ but higher than that for LyC $\left(\sim 10^{17} \mathrm{~cm}^{-1}\right)$.

\section{Outlook}

Detailed studies of local galaxies are now being used by several research groups to understand the ionising budget and the potential leakage of $\mathrm{LyC}$ photons. Another avenue is at $z=2.2-2.4$ where $\mathrm{H} \alpha, \mathrm{H} \beta$ and e.g. oxygen ionic transitions can be be observed from the ground combined with observations of LyC photons with HST/UVIS in the near UV, to directly constrain the LyC leakage from deep field samples.

\section{References}

Leitet, Bergvall, Hayes et al. 2014, A\&A, 553, 106

Leitherer, Ekström, Meynet et al. 2012, ApJS, 212, 14

Östlin, G., Hayes, M., Duval, F., et al. 2014, ApJ, 797, 11

Pellegrini, Oey, Winkler et al. 2012, ApJ, 755, 40

Verhamme, Orlitova, Schaerer, Hayes 2015, A\&SA, 578, 7 